\title{
Survival outcomes of hepatic resection compared with transarterial chemoembolization or sorafenib for hepatocellular carcinoma with portal vein tumor thrombosis
}

Jung Min Lee', Byoung Kuk Jang ${ }^{1}$, Yoo Jin Lee', Wang Yong Choi', Sei Myong Choi', Woo Jin Chung ${ }^{1}$, Jae Seok Hwang ${ }^{1}$, Koo Jeong Kang ${ }^{2}$, Young Hwan Kim ${ }^{3}$, Anil Kumar Chauhan ${ }^{4}$, Soo Young Park ${ }^{5}$, Won Young Tak ${ }^{5}$, Young Oh Kweon ${ }^{5}$, Byung Seok Kim ${ }^{6}$, and Chang Hyeong Lee ${ }^{6}$

Departments of ${ }^{1}$ Internal Medicine, ${ }^{2}$ Surgery, ${ }^{3}$ Radiology, and ${ }^{4}$ Immunology, Keimyung University School of Medicine; ${ }^{5}$ Department of Internal Medicine, Kyungpook National University School of Medicine; ${ }^{6}$ Department of Internal Medicine, Catholic University of Daegu School of Medicine, Daegu, Korea

Background/Aims: Treating hepatocellular carcinoma (HCC) with portal vein tumor thrombosis (PVTT) remains controversial. We compared the outcomes of hepatic resection (HR), transarterial chemoembolization (TACE), and sorafenib therapy as treatments for HCC with PVTT.

Methods: Patients diagnosed as HCC with PVTT between January 2000 and December 2011 who received treatment with sorafenib, HR, or TACE were included. Patients with main PVTT, superior mesenteric vein tumor thrombosis, or ChildTurcotte-Pugh (CTP) class C were excluded. The records of 172 patients were analyzed retrospectively. HR, TACE, and sorafenib treatment were performed is 40,80 , and 52 patients respectively. PVTT was classified as either involving the segmental branch (type I) or extending to involve the right or left portal vein (type II).

Results: The median survival time was significantly longer in the HR group (19.9 months) than in the TACE and sorafenib groups ( 6.6 and 6.2 months, respectively; both $P<0.001$ ), and did not differ significantly between the latter two groups $(\mathrm{P}=0.698)$. Among patients with CTP class A, type I PVTT or unilobar-involved HCC, the median survival time was longer in the HR group than in the TACE and sorafenib groups $(P=0.006)$. In univariate analyses, the initial treatment method, tumor size, PVTT type, involved lobe, CTP class, and presence of cirrhosis or ascites were correlated with overall survival. The significant prognostic factors for overall survival in Cox proportional-hazards regression analysis were initial treatment method (HR vs. TACE: hazard ratio $=1.750, P=0.036$; HR vs. sorafenib: hazard ratio $=2.262, P=0.006$ ), involved lobe (hazard ratio $=1.705, P=0.008$ ), PVTT type (hazard ratio $=1.617, P=0.013$ ), and CTP class (hazard ratio $=1.712, P=0.012$ ).

Conclusions: Compared with TACE or sorafenib, HR may prolong the survival of patients with HCC in cases of CTP class A, type I PVTT or unilobar-involved HCC. (Clin Mol Hepatol 2016;22:160-167)

Keywords: Hepatocellular carcinoma; Portal vein tumor thrombosis; Transarterial chemoembolization; Hepatic resection; Sorafenib

\footnotetext{
Abbreviations:

AFP, alpha-fetoprotein; ALT, alanine aminotransferase; AST, aspartate aminotransferase; BCLC, Barcelona Clinic Liver Cancer; $\mathrm{CT}$, computed tomography; CTP, Child-Turcotte-Pugh; HBV, hepatitis B virus; HCC, hepatocellular carcinoma; $\mathrm{HCV}$, hepatitis $\mathrm{C}$ virus; $\mathrm{HR}$, hepatic resection; MELD, model for end-stage liver disease; PIVKA-II, protein induced by vitamin Kabsence or antagonist-II; PT, prothrombin time; PVTT, portal vein tumor thrombosis; RFA, radiofrequency ablation; TACE, transarterial chemoembolization
}

Corresponding author : Byoung Kuk Jang

Department of Internal Medicine, Keimyung University Dongsan Hospital, 56 Dalsung-ro, Jung-gu, Daegu 41931, Korea Tel: +82-53-250-7088, Fax: +82-53-250-7422

E-mail: jangha106@dsmc.or.kr 


\section{INTRODUCTION}

Hepatocellular carcinoma (HCC) has one of the highest incidence rates of all malignancies worldwide. Incidence rates of HCC in Asian and African countries are generally higher than those in Western countries because the prevalence of chronic hepatitis $B$ infection is generally higher in Asia and Africa. ${ }^{1}$ For early detection of HCC, liver imaging and assessments of alpha-fetoprotein (AFP) levels have been recommended for patients with underlying viral liver disease. ${ }^{2}$ Nevertheless, many patients are diagnosed as a cancer at the advanced stage. ${ }^{1,2}$ Portal vein tumor thrombosis (PVTT) is a poor prognostic factor detected in $20-60 \%$ of HCC cases. The median survival time among patients with HCC with PVTT has been reported as 2.7-4.0 months without intervention. $^{3-6}$ In clinical practice, however, the treatment of patients with HCC with PVTT is complicated and controversial. ${ }^{7,8}$ As per the Barcelona Clinic Liver Cancer (BCLC) guidelines, HCC with PVTT is classified as stage $C$ (advanced stage) and sorafenib is recommended for treatment. ${ }^{7}$ However, various treatments have been applied to improve the prognosis of patients with HCC with PVTT in a real clinical field. According to the National Survey for Primary Liver Cancer in Japan, the survival of patients with HCC with PVTT can be improved by some therapeutic modalities, including surgery, radiation therapy, transarterial chemoembolization (TACE), hepatic arterial infusion chemotherapy (HAIC), and combined treatments. ${ }^{9}$ However, the available data on patients with HCC with PVTT have generally been insufficient for comparisons of the survival outcomes associated with different treatment modalities. Although the recommended treatment, sorafenib, was associated with superior outcomes when compared with best supportive care, ${ }^{10}$ no studies have compared sorafenib with other treatments, such as TACE, radiation or surgery.

The aim of this study was to retrospectively compare the survival outcomes of patients with HCC with PVTT after treatment with hepatic resection (HR), TACE or sorafenib. In addition, we sought to identify factors that influenced survival outcomes.

\section{MATERIALS AND METHODS}

\section{Study Design}

Patients who received HR, TACE, or sorafenib as an initial treatment for HCC with PVTT at any of the three tertiary university hospitals between January 2000 and December 2011 were select- ed for this retrospective study. PVTT was classified according to 4 types, as described by Shi et al. ${ }^{11}$ Type I PVTT is defined as tumor thrombi involving the segmental branches of the portal vein or above. Type II PVTT is defined as tumor thrombi extending to involve the right/left portal vein. Type III PVTT is defined as thrombi involving the main portal vein. Type IV PVTT is defined as thrombi involving the superior mesenteric vein. Cases involving type III or type IV PVTT were excluded because HR and TACE are rarely applied in these cases. Accordingly, the current study only included cases of HCC with type I or type II PVTT.

\section{Patients}

The European Association for the Study of the Liver guideline was used for diagnosis of HCC. All HR cases were confirmed by histological review. Patients were enrolled according to the following inclusion criteria: 1) a case of HCC with no previous treatment, 2) presence of PVTT on imaging studies, and 3) the size of the main HCC lesion was less than $10 \mathrm{~cm}$. Patients who satisfied any of the following criteria were excluded: 1) presence of extrahepatic spreading, 2) the Child-Turcotte-Pugh (CTP) class C, or 3) the case involved type III or type IV PVTT. The technique of arterial embolization was administered as the standard TACE procedure. After tumor stain, Doxorubicin was infused maximally 50 mg. Hepatic angiography to confirm occlusion of the tumor feeding artery and to look for another residual vascular tumor blush as well. The following parameters were analyzed as potential predators of survival, age, gender, liver cirrhosis status, liver function status, CTP class, Model For End-Stage Liver Disease (MELD) score, platelet count, prothrombin time (PT), total bilirubin level, alanine aminotransferase (ALT) level, aspartate aminotransferase (AST) level, albumin level, and etiology of liver disease.

\section{Follow-up}

In the HR group, the first follow-up was 3 weeks after HR. In the TACE group, the first follow-up was performed 3 weeks after treatment. The patients were monitored for tumor recurrence or progression on the basis of AFP levels as well as contrast computed tomography (CT) or magnetic resonance imaging findings. Thereafter, contrast CT scans were performed once every 3 months for surveillance until disease progression in the $H R$ and TACE groups. In the sorafenib group, contrast CT was performed once every 6 weeks for the same purpose. On each of the followup days, each patient underwent blood tests, including blood cell 
counts and liver function tests. In the TACE group, additional TACE sessions were performed every 4 weeks until the tumor was completely ablated or the tumor had progressed. For patients who developed cancer recurrence after HR, adequate local treatments were applied, such as TACE, radiofrequency ablation (RFA), or reoperation. Patients who showed no response or progression after sorafenib treatment received best supportive care or another local treatment.

\section{Statistical Analyses}

SPSS 19 statistical software (SPSS Inc., Chicago, IL, USA) was used for data analysis. Overall survival was calculated using the life-table method. For univariate analyses, the ANOVA test was used for continuous data and the chi square test and two tailed Fisher exact test for categorical data. Survival curves were estimated using the Kaplan-Meier method and the differences between groups were evaluated by log-rank test. Prognostic relevance was evaluated by Cox's proportion hazard regression

Table 1. Patient characteristics at baseline

\begin{tabular}{|c|c|c|c|c|}
\hline & $\mathrm{HR}(\mathrm{n}=40)$ & TACE $(n=80)$ & Sorafenib $(n=52)$ & $P$-value \\
\hline Age, y (mean $\pm S D)$ & $55.0 \pm 12.9$ & $58.3 \pm 10.5$ & $57.3 \pm 12.4$ & 0.348 \\
\hline $\operatorname{Sex}(M / F)$ & $30 / 10$ & $67 / 13$ & $44 / 8$ & 0.420 \\
\hline Cirrhosis, no. (\%) & $27(67.5)$ & $73(91.3)$ & $51(98.1)$ & $<0.001$ \\
\hline Etiology & & & & 0.116 \\
\hline HBV & 31 & 54 & 39 & \\
\hline $\mathrm{HCV}$ & 4 & 8 & 2 & \\
\hline Alcohol & 3 & 11 & 11 & \\
\hline Unkown & 2 & 7 & 0 & \\
\hline CTP class, no. (\%) & & & & 0.059 \\
\hline A & $35(87.5)$ & $58(72.5)$ & $45(86.5)$ & \\
\hline B & $5(12.5)$ & $22(27.5)$ & $7(13.5)$ & \\
\hline Ascites, no. (\%) & $2(5.0)$ & $27(33.8)$ & $9(17.3)$ & 0.001 \\
\hline Tumor size, cm, no. (\%) & & & & $<0.001$ \\
\hline$\leqq 5$ & $20(50.0)$ & $10(12.5)$ & $8(15.4)$ & \\
\hline$>5$ & $20(50.0)$ & $70(87.5)$ & $44(84.6)$ & \\
\hline PVTT site, no. (\%) & & & & 0.002 \\
\hline Segmental & $26(65.0)$ & $31(38.8)$ & $16(30.8)$ & \\
\hline Lobar & $14(35.0)$ & $49(61.3))$ & $36(69.2)$ & \\
\hline Lobe, no. (\%) & & & & $<0.001$ \\
\hline Unilobe & $39(97.5)$ & $44(55.0)$ & $39(75.0)$ & \\
\hline Bilobe & $1(2.5)$ & $36(45.0)$ & $13(25.0)$ & \\
\hline AFP $(n g / m L$, mean $\pm S D)$ & $10,728 \pm 25,073$ & $27,302 \pm 71,902$ & $16,663 \pm 35,668$ & 0.266 \\
\hline PIVKA-II (mAU/mL, mean $\pm S D)$ & $4,236 \pm 13,166$ & $13,348 \pm 34,992$ & $7,411 \pm 24,758$ & 0.038 \\
\hline PT (sec, mean \pm SD) & $12.1 \pm 1.7$ & $12.8 \pm 1.8$ & $12.5 \pm 1.3$ & 0.072 \\
\hline Total bilirubin $(T)(\mathrm{mg} / \mathrm{dL}$, mean $\pm S D)$ & $0.9 \pm 0.9$ & $1.3 \pm 1.3$ & $1.2 \pm 0.7$ & 0.162 \\
\hline AST (IU/L, mean $\pm S D)$ & $77.0 \pm 81.0$ & $95.2 \pm 83.2$ & $112.1 \pm 106.4$ & 0.184 \\
\hline ALT (IU/L, mean $\pm S D)$ & $48.9 \pm 60.1$ & $57.8 \pm 53.0$ & $42.2 \pm 28.5$ & 0.192 \\
\hline Albumin (g/dL, mean $\pm S D)$ & $4.0 \pm 0.6$ & $3.6 \pm 0.5$ & $3.5 \pm 0.6$ & $<0.001$ \\
\hline MELD score (mean \pm SD) & $8.3 \pm 3.0$ & $9.2 \pm 2.7$ & $8.9 \pm 2.1$ & 0.198 \\
\hline
\end{tabular}

HR, hepatic resection; TACE, transarterial chemoembolization; HBV, hepatitis B virus; HCV, hepatitis C virus; CTP, Child-Turcotte-Pugh; PVTT, portal vein tumor thrombosis; AFP, $\alpha$-fetoprotein; PIVKA-II, protein induced by vitamin K absence or antagonist-II; PT, prothrombin time; AST, aspartate aminotransferase; ALT, alanine aminotransferase; MELD, model for end-stage liver disease. 
analysis. Values of $P<0.05$ were considered significant.

\section{Ethics statement}

The study protocol was approved by the Institutional Review Board of Keimyung University Dongsan Hospital.

\section{RESULTS}

\section{Patient characteristics at baseline}

A total of 172 patients with HCC with PVTT were included in our analysis; 40 patients underwent HR, 80 patients underwent TACE, and 52 patients received sorafenib therapy as the initial treatment. The clinical characteristics of the three groups of pa-
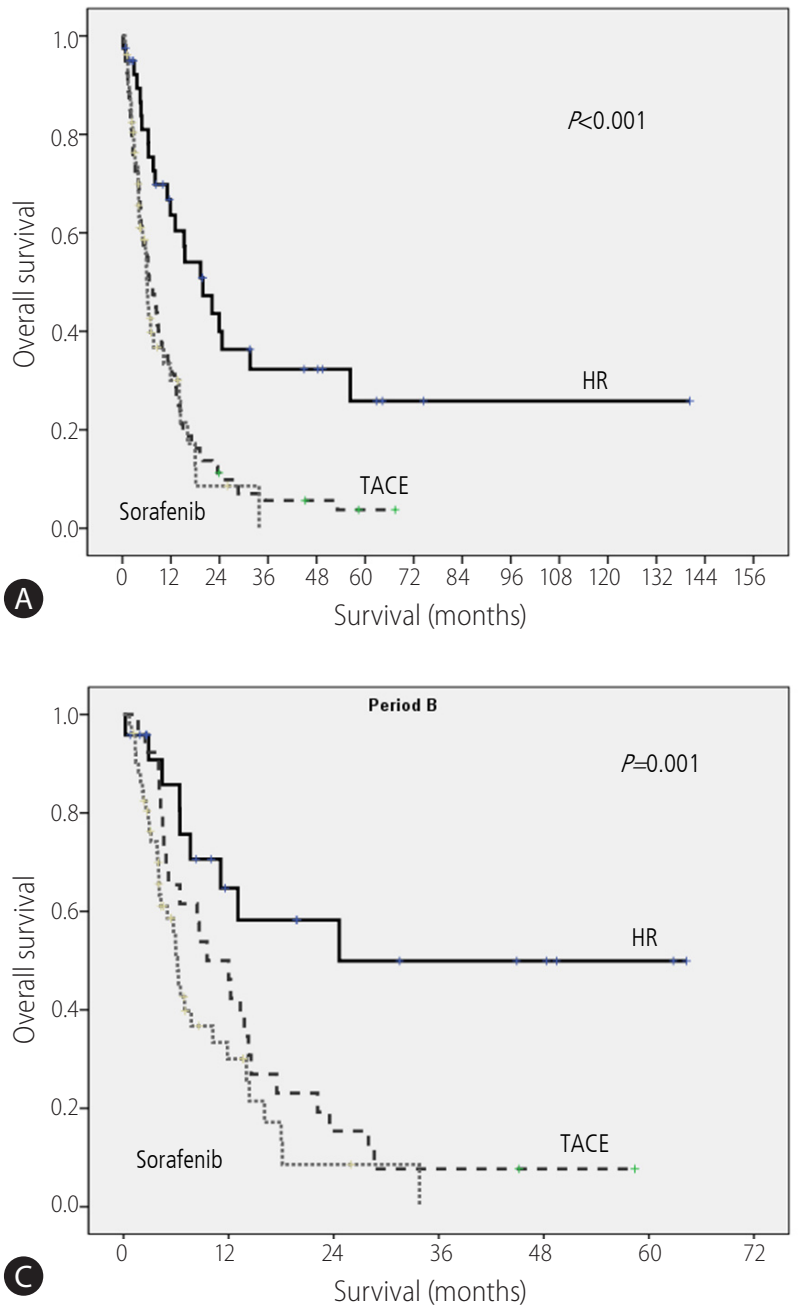

tients are summarized in Table 1. The median ages in the HR, TACE, and sorafenib groups were 55.0, 58.3, and 57.3 years, respectively. There were no significant differences at baseline in terms of age, sex, or CTP class between the groups. Cirrhosis (as clearly proved by imaging) was present in 68\% (28/40 patients) of the HR group, $91 \%$ (73/80 patients) of the TACE group, and $98 \%$ (51/52 patients) of the sorafenib group, constituting a significant difference $(P<0.001)$. There are also significant differences in terms of the tumor size, PVTT site, and lobe between the groups $(P<0.001, P=0.002, P<0.001)$. However, no significant differences in terms of hepatic function scores, including the CTP class and MELD scores, were observed between the groups. In addition, no significant difference in platelet count, PT, bilirubin level, etiology of liver disease, and other hepatic function markers was observed between the three groups.

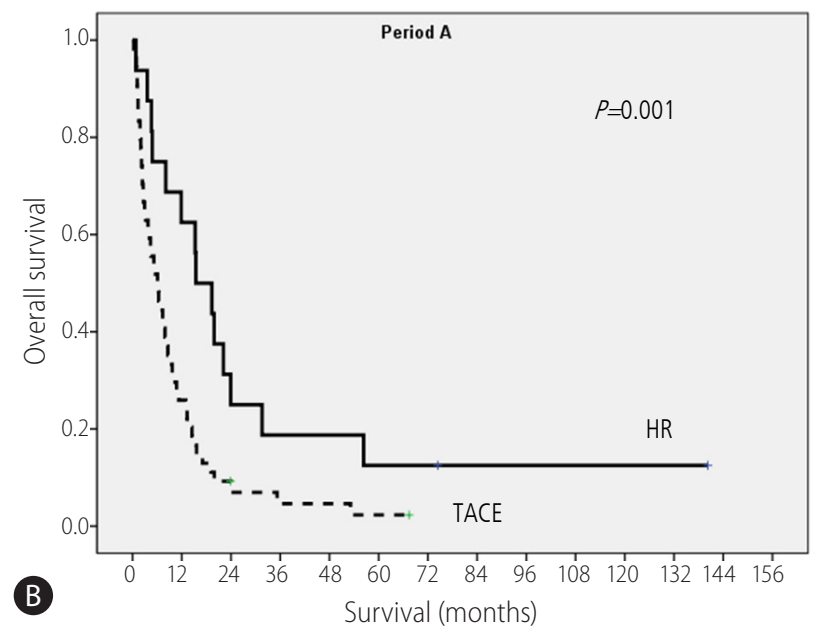

Figure 1. Overall survival curves for the HR, TACE, and sorafenib groups (A), for the HR and TACE groups in period A (January 2000 to December 2007) (B), and for the HR, TACE, and sorafenib groups in period B (January 2008 to December 2011) (C). HR, hepatic resection; TACE, transarterial chemoembolization. 


\section{Survival analyses}

The median survival times in the HR, TACE, and sorafenib groups were 19.9, 6.6, and 6.2 months, respectively $(P<0.001$, Fig. $1 A)$. The HR group had significantly longer median survival period than the TACE and sorafenib groups $(P<0.001$ and $P<0.001)$. However, there was no significant difference between the median survival times of the TACE and sorafenib groups ( $P=0.698)$.

In consideration of the approval date for sorafenib in South Korea, we performed an additional analysis that was stratified into two periods: January 2000 to December 2007 (period A) and January 2008 to December 2011 (period B). In period A, the median survival times in the HR and TACE groups were 15.4 months and 6.1 months, respectively ( $P=0.007$; Fig. 1B). In period $B$, the median survival times in the HR, TACE, and sorafenib groups were 24.6 months, 9.5 months, and 6.2 months, respectively $(P=0.001$; Fig. 1C). As the result of total period, the median survival time of the HR group was significantly longer than that of the TACE and sorafenib groups in period $B(P=0.001$ and $P=0.001$ ). The median survival times of the TACE group also did not differ significantly from those of the sorafenib group $(P=0.148)$.

\section{Subgroup analyses}

In a subgroup analysis limited to patients with CTP class A during period $B$, overall survival was significantly longer in the $H R$ group (24.6 months) than in the TACE group or sorafenib group (12.0 months and 6.5 months, $P=0.030$ and $P=0.001$ ). However, the median survival times did not differ significantly between the
TACE and sorafenib groups $(P=0.203)$. For patients with CTP class $B$ during period $B$, there was no difference in the median survival times between three groups $(P=0.123)$. For patients with type I PVTT during period $B$, the median survival times were significantly different between the HR, TACE, and sorafenib groups $(P=0.003$; Fig. 2A). A significant difference in median survival times was observed only between the HR and sorafenib groups with type I PVTT $(P=0.002)$. However, median survival times between the HR and TACE groups and between the TACE and sorafenib groups were not significantly different $(P=0.069$ and $P=0.087$ ). For type II PVTT during period $B$, however, there were no significant differences in median survival times between three groups ( $P=0.499$; Fig. $2 \mathrm{~B}$ ). In a subgroup analysis of patients with tumors $<5 \mathrm{~cm}$ in size in period $B$, no significant difference in overall survival was observed between three groups $(P=0.307)$. However, among patients with tumors $>5 \mathrm{~cm}$ in size in period $B$, overall survival times were significantly longer in the HR group (24.6 months) than in the TACE and sorafenib groups (9.5 months and 5.9 months, $P=0.038$ and $P=0.007$ ). Among patients without ascites, the HR group had significantly better overall survival than the TACE or sorafenib groups $(P=0.001)$. In a subgroup analysis limited to patients with CTP class A, type I PVTT and HCC in unilobe, the HR group had significantly longer overall survival (23.9 months) than the sorafenib group (11.9 months, $P=0.006)$. However, median survival times of the HR group did not differ from those of the TACE group (14.5 months, $P=0.082$ ). Treatment-specific 1-, 2-, and 3-year survival rates during period $B$ are shown in Table 2.
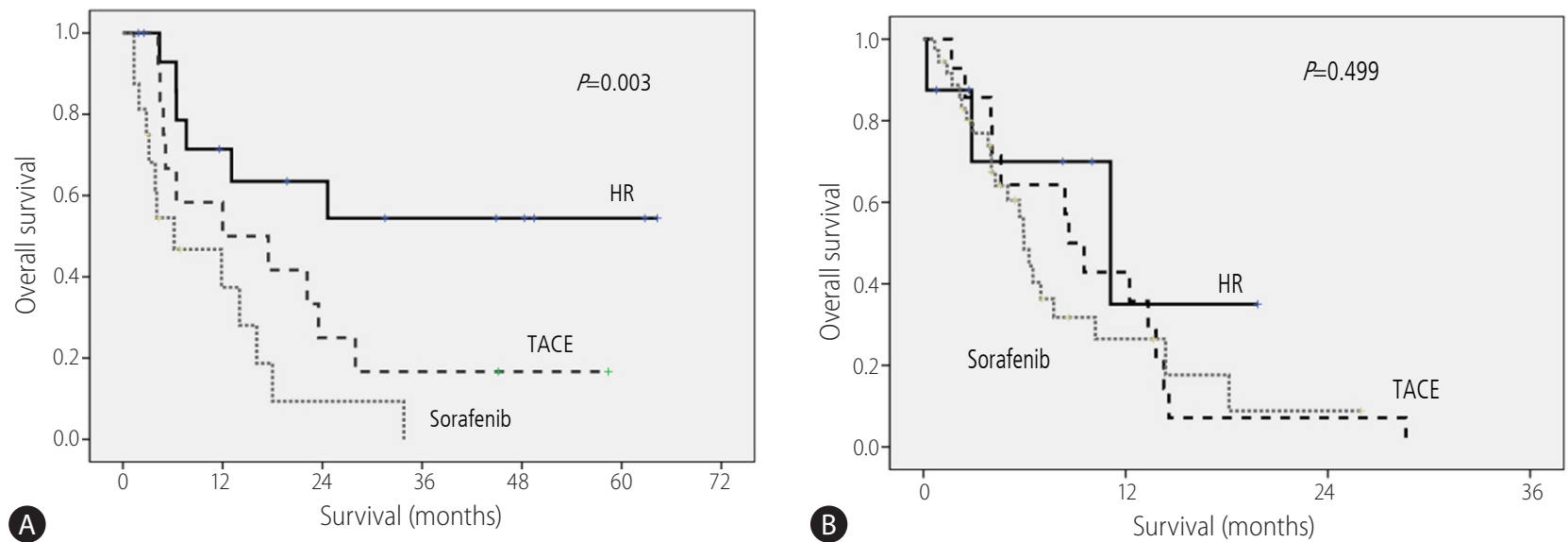

Figure 2. Overall survival curves for patients with type I PVTT who received HR, TACE, or sorafenib in period B (January 2008 to December 2011 ) (A) and for patients with type II PVTT in period B (B). HR, hepatic resection; TACE, transarterial chemoembolization; PVTT, portal vein tumor thrombosis. 
Table 2. Comparison of overall survival rates according to treatment method in period $B$

\begin{tabular}{|c|c|c|c|c|c|c|}
\hline \multirow{2}{*}{ Treatment by tumor type } & & \multirow{2}{*}{ Patients no. } & \multicolumn{4}{|c|}{ Survival rate (\%) } \\
\hline & & & 1 year & 2 year & 3 year & $P$-value \\
\hline \multirow[t]{3}{*}{ All HCC } & $H R$ & 24 & 64.7 & 58.3 & 49.9 & $<0.001$ \\
\hline & TACE & 26 & 46.2 & 15.4 & 7.7 & \\
\hline & Sorafenib & 52 & 30.0 & 8.6 & 0 & \\
\hline \multicolumn{7}{|l|}{ CTP class } \\
\hline \multirow[t]{3}{*}{ A } & $H R$ & 21 & 65.7 & 58.4 & 48.6 & 0.004 \\
\hline & TACE & 20 & 50.0 & 15.0 & 10.0 & \\
\hline & Sorafenib & 45 & 33.5 & 9.6 & 0 & \\
\hline \multirow[t]{3}{*}{ B } & $H R$ & 3 & 66.7 & 66.7 & 66.7 & 0.124 \\
\hline & TACE & 6 & 33.3 & 16.7 & 0 & \\
\hline & Sorafenib & 7 & 0 & 0 & 0 & \\
\hline \multicolumn{7}{|l|}{ PVTT site } \\
\hline \multirow[t]{3}{*}{ Type I PVTT } & $H R$ & 16 & 71.4 & 54.4 & 54.4 & 0.003 \\
\hline & TACE & 12 & 50.0 & 25.0 & 16.7 & \\
\hline & Sorafenib & 16 & 37.4 & 9.4 & 0 & \\
\hline \multirow[t]{3}{*}{ Type II PVTT } & $H R$ & 8 & 35.0 & 0 & 0 & 0.499 \\
\hline & TACE & 14 & 35.7 & 7.1 & 0 & \\
\hline & Sorafenib & 36 & 26.5 & 8.8 & 0 & \\
\hline \multicolumn{7}{|l|}{ Size } \\
\hline \multirow[t]{3}{*}{$\leq 5 \mathrm{~cm}$} & $H R$ & 14 & 65.0 & 52.0 & 52.0 & 0.307 \\
\hline & TACE & 3 & 66.7 & 0 & 0 & \\
\hline & Sorafenib & 8 & 57.1 & 14.3 & 0 & \\
\hline \multirow[t]{3}{*}{$>5 \mathrm{~cm}$} & $H R$ & 10 & 64.8 & 64.8 & 48.6 & 0.005 \\
\hline & TACE & 23 & 43.5 & 17.4 & 8.7 & \\
\hline & Sorafenib & 44 & 23.2 & 7.7 & 0 & \\
\hline \multicolumn{7}{|l|}{ Lobe } \\
\hline \multirow[t]{3}{*}{ Unilobe } & $H R$ & 25 & 67.5 & 60.8 & 52.1 & 0.001 \\
\hline & TACE & 15 & 60.0 & 20.0 & 6.7 & \\
\hline & Sorafenib & 39 & 31.8 & 10.6 & 0 & \\
\hline \multirow[t]{3}{*}{ Bilobe } & $H R$ & 1 & 0 & 0 & 0 & $<0.001$ \\
\hline & TACE & 10 & 27.3 & 9.1 & 9.1 & \\
\hline & Sorafenib & 13 & 20.8 & 0 & 0 & \\
\hline
\end{tabular}

HCC, hepatocellular carcinoma; HR, hepatic resection; TACE, transarterial chemoembolization; CTP, Child-Turcotte-Pugh; PVTT, portal vein tumor thrombosis.

\section{Univariate and multivariate analyses of overall survival for all patients}

Between overall survival and 20 variables with known values for all patients were evaluated by univariate analysis. In the univariate analyses, initial treatment method, tumor size, PVTT type, lobe, CTP class, and presence of cirrhosis or ascites were correlated with overall survival. In multivariate Cox proportional hazards regression analysis, initial treatment method (HR vs. TACE hazard ratio $=1.750 ; 95 \%$ confidence interval $[\mathrm{Cl}], 1.037-2.953 ; P=0.036$, HR vs. sorafenib hazard ratio $=2.262 ; 95 \% \mathrm{Cl}, 1.270-4.027$; $P=0.006$ ), lobe (hazard ratio=1.705; $95 \% \mathrm{Cl}, 1.147-2.535$; $P=0.008$ ), PVTT type (hazard ratio=1.617; $95 \% \mathrm{Cl}, 1.108-2.359$; $P=0.013$ ), and CTP class (hazard ratio $=1.712 ; 95 \% \mathrm{Cl}, 1.125$ 2.607; $P=0.012$ ) were significant prognostic factors for overall survival (Table 3). 
Table 3. Multivariate analysis of the overall survival for all patients

\begin{tabular}{lccc}
\hline Variables & Hazard ratio & $\mathbf{9 5 \%} \mathbf{C l}$ & P-value \\
\hline Treatment & & & \\
\hline HR vs. TACE & 1.750 & $1.037-2.953$ & 0.036 \\
\hline HR vs. Sorafenib & 2.262 & $1.270-4.027$ & 0.006 \\
Lobe & 1.705 & $1.147-2.535$ & 0.008 \\
PVTT site & 1.617 & $1.108-2.359$ & 0.013 \\
CTP class & 1.712 & $1.125-2.607$ & 0.012 \\
\hline
\end{tabular}

$\mathrm{Cl}$, confidence interval; HR, hepatic resection; TACE, transarterial chemoembolization; PVTT, portal vein tumor thrombosis; CTP, Child-Turcotte-Pugh.

\section{DISCUSSION}

According to the BCLC staging system, chemotherapy with a molecular-targeted agent is the only treatment option for patients with advanced HCC. In 2008, a large randomized, controlled study showed that patients with advanced HCC who received sorafenib treatment had a median survival benefit of approximately 3 months, as compared with the placebo group..$^{10}$ Other studies have shown that TACE is more effective than best supportive care, even though the outcomes of TACE remain poor. ${ }^{12-15}$ However, the treatment of locally advanced HCC remains controversial. In Asian countries, various treatment methods have been attempted. The Asian Pacific Association for the Study of the Liver and the Japan Society of Hepatology recommended TACE, HAIC, ablation, or surgical treatment for locally advanced HCC. ${ }^{16,17}$ Peng et al. ${ }^{18}{ }^{18}$ who conducted a retrospective study of patients with HCC with PVTT comparing HR and TACE, concluded that HR provided a survival benefit for patients with resectable HCC with PVTT. Shi et al. also suggested that HR was associated with better clinical results than TACE for the treatment of HCC with PVTT."

In our study, sorafenib (the standard of care for patients with HCC with PVTT) was compared with HR and TACE. As previously reported, HR was associated with a longer median survival time and greater overall survival rates than sorafenib or TACE. The outcomes of HR were most notably superior to the outcomes of TACE or sorafenib for advanced HCC patients with good hepatic function, CTP class A and type I PVTT. Despite significant differences between the three groups in terms of albumin levels at baseline, the levels were within the normal range. At baseline, significant differences were also observed for other variables, including ascites status and liver cirrhosis status. However, there were no significant differences in MELD scores and CTP classes. We performed subgroup analyses of patients without ascites and patients belonging to CTP class A. In both of these subgroups, HR was associated with significantly better survival than TACE or sorafenib. Accordingly, we believe that the differences in baseline laboratory characteristics and liver cirrhosis did not influence our assessment of effectiveness. In addition, the presence of cirrhosis has not been included as a meaningful factor in any guideline's treatment algorithm for patients with HCC. Because sorafenib was approved in South Korea partway through the study period, we divided our enrollment period into two periods: A and B. We found that HR was associated with superior survival in both peri$\operatorname{od} A$ and period $B$. However, in the HR group, the median survival time was 15.4 months in period $A$ and 24.6 months in period $B$, and in the TACE group, the median survival time was and 6.1 months in period $A$ and 9.5 months in period $B$. These results may be explained by advancements in TACE, operative techniques, and bedside care. Recently, there have been further developments in the treatment methods and techniques for HCC. Therefore, it is debatable whether sorafenib alone is the best choice for patients with advanced-stage $\mathrm{HCC}$, according to the BCLC. There are some limitations to the current study. First, it is a retrospective design. Second, there may be selection bias because patients with relatively good hepatic function and easy-to-resection HCC might be included in the HR group. Third, the sample size was small and limited to South Korea. Because of differences in underlying liver diseases, our results may not be applicable to patients with HCC with PVTT in other countries. Fourth, portal hypertension and the indocyanine green clearance level were not assessed in all of the enrolled patients, although these factors are strongly associated with prognosis. Fifth, TACE, RFA, HAIC, operations, moleculartargeted agents, and other treatment modalities were applied for recurrent or remnant tumor after initial treatment.

Despite these limitations, this retrospective study indicated that $H R$, as a first treatment option, may provide better long term survival than TACE or sorafenib for resectable HCC patients with type I PVTT and good hepatic function. Therefore, we suggest that treatment strategies for HCC with PVTT should not be limited to sorafenib. To prolong the survival of patients with advanced BCLC 
stage HCC, the selection of treatment method should be considered according to CTP class, the extent of PVTT, and location of lesion.

\section{Acknowledgments}

This work was supported by the National Research Foundation of Korea (NRF) Grant funded by the Korean Government (MSIP) (No. 2014R1A5A2010008).

\section{Conflicts of Interest}

The authors have no conflicts to disclose.

\section{REFERENCES}

1. Yang JD, Roberts LR. Hepatocellular carcinoma: A global view. Nat Rev Gastroenterol Hepatol 2010;7:448-458.

2. Cheung TK, Lai CL, Wong BC, Fung J, Yuen MF. Clinical features, biochemical parameters, and virological profiles of patients with hepatocellular carcinoma in Hong Kong. Aliment Pharmacol Ther 2006;24:573-583.

3. Llovet JM, Burroughs A, Bruix J. Hepatocellular carcinoma. Lancet 2003;362:1907-1917.

4. Llovet JM, Bustamante J, Castells A, Vilana R, Ayuso Mdel C, Sala M, et al. Natural history of untreated nonsurgical hepatocellular carcinoma: rationale for the design and evaluation of therapeutic trials. Hepatology 1999;29:62-67.

5. Villa E, Moles A, Ferretti I, Buttafoco P, Grottola A, Del Buono M, et al. Natural history of inoperable hepatocellular carcinoma: estrogen receptors' status in the tumor is the strongest prognostic factor for survival. Hepatology 2000;32:233-238.

6. Lai EC, Fan ST, Lo CM, Chu KM, Liu CL, Wong J. Hepatic resection for hepatocellular carcinoma. An audit of 343 patients. Ann Surg 1995;221:291-298.

7. Bruix J, Sherman $M$; American Association for the Study of Liver Diseases(AASLD). Management of hepatocellular carcinoma: an update. Hepatology 2011;53:1020-1022.

8. European Association For The Study Of The Liver(EASL), European Organisation For Research And Treatment Of Cancer(EORTC). EASLEORTC clinical practice guidelines: management of hepatocellular carcinoma. J Hepatol 2012;56:908-943.

9. Minagawa M, Makuuchi M. Treatment of hepatocellular carcinoma accompanied by portal vein tumor thrombus. World J Gastroenterol 2006;12:7561-7567.

10. Llovet JM, Ricci S, Mazzaferro V, Hilgard P, Gane E, Blanc JF, et al. Sorafenib in advanced hepatocellular carcinoma. N Engl J Med 2008;359:378-390.

11. Shi J, Lai EC, Li N, Guo WX, Xue J, Lau WY, et al. Surgical treatment of hepatocellular carcinoma with portal vein tumor thrombus. Ann Surg Oncol 2010;17:2073-2080.

12. Lee HS, Kim JS, Choi IJ, Chung JW, Park JH, Kim CY. The safety and efficacy of transcatheter arterial chemoembolization in the treatment of patients with hepatocellular carcinoma and main portal vein obstruction. A prospective controlled study. Cancer 1997;79:20872094.

13. Luo J, Guo RP, Lai EC, Zhang YJ, Lau WY, Chen MS, et al. Transarterial chemoembolization for unresectable hepatocellular carcinoma with portal vein tumor thrombosis: a prospective comparative study. Ann Surg Oncol 2011;18:413-420.

14. Takayasu K, Arii S, Ikai I, Omata M, Okita K, Ichida T, et al. Prospective cohort study of transarterial chemoembolization for unresectable hepatocellular carcinoma in 8510 patients. Gastroenterology 2006;131:461-469.

15. Kim KM, Kim JH, Park IS, Ko GY, Yoon HK, Sung KB, et al. Reappraisal of repeated transarterial chemoembolization in the treatment of hepatocellular carcinoma with portal vein invasion. J Gastroenterol Hepatol 2009;24:806-814.

16. Omata M, Lesmana LA, Tateishi R, Chen PJ, Lin SM, Yoshida H, et al. Asian Pacific Association for the Study of the Liver consensus recommendations on hepatocellular carcinoma. Hepatol Int 2010;4:439474.

17. Kudo M, Izumi N, Kokudo N, Matsui O, Sakamoto M, Nakashima O, et al. Management of hepatocellular carcinoma in Japan: Consensus-Based Clinical Practice Guidelines proposed by the Japan Society of Hepatology (JSH) 2010 updated version. Dig Dis 2011;29:339364.

18. Peng ZW, Guo RP, Zhang YJ, Lin XJ, Chen MS, Lau WY. Hepatic resection versus transcatheter arterial chemoembolization for the treatment of hepatocellular carcinoma with portal vein tumor thrombus. Cancer 2012;118:4725-4736. 